Appl. Set-Valued Anal. Optim. 2 (2020), No. 1, pp. 95-107

Available online at http://asvao.biemdas.com

https://doi.org/10.23952/asvao.2.2020.1.06

\title{
AN IMPROVEMENT LQP METHOD FOR NONLINEAR COMPLEMENTARITY PROBLEMS
}

\author{
ABDELLAH BNOUHACHEM ${ }^{1}$, XIAOLONG QIN ${ }^{2, *}$ \\ ${ }^{1}$ Equipe MAISI, Ibn Zohr University, ENSA, BP 1136, Agadir, Morocco \\ ${ }^{2}$ General Education Center, National Yunlin University of Science and Technology, Douliou 64002, Taiwan
}

\begin{abstract}
In this paper, we propose a new modified logarithmic-quadratic proximal method for solving the nonlinear complementarity problem. An easily measurable error term is proposed with further relaxed error bound and a new step length is employed to reach substantial progress in each iteration. Preliminary numerical experiments are included to illustrate the advantage and efficiency of the proposed method.
\end{abstract}

Keywords. Nonlinear complementarity problems; Pseudomonotone operators; Interior proximal methods.

\section{INTRODUCTION}

The nonlinear complementarity problem (NCP) is to determine a vector $x \in \mathbb{R}^{n}$ such that

$$
x \geq 0, \quad F(x) \geq 0 \quad \text { and } \quad x^{T} F(x)=0,
$$

where $F$ is a nonlinear mapping from $\mathbb{R}^{n}$ into itself. Recently, various iterative methods, which are powerful techniques for solving the NCP effectively, are under the spotlight of researchers; see, e.g., $[2,3,8,9,10,11,12,13,14,15,17,20,25,26]$ and the references therein.

In the sequel, we make the following assumptions that $F$ is continuous and pseudomonotone with respect to $\mathbb{R}_{+}^{n}$ and the solution set of (1.1), denoted by $\Omega^{*}$, is nonempty.

The proximal point algorithm (PPA) suggested by Martinet [18] is one of the most effective iterative methods for finding solutions of the NCP associated with finding a zero of the maximal monotone operator $T(x):=F(x)+N_{\mathbb{R}_{+}^{n}}(x)$, where $N_{\mathbb{R}_{+}^{n}}($.$) is the normal cone operator to \mathbb{R}_{+}^{n}$ defined by

$$
N_{\mathbb{R}_{+}^{n}}(x)=\left\{\begin{array}{l}
\left\{y \in \mathbb{R}^{n}: y^{T}(v-x) \leq 0, \quad \forall v \in \mathbb{R}_{+}^{n}\right\}, \quad \text { if } \begin{array}{r}
x \in \mathbb{R}_{+}^{n}, \\
\emptyset,
\end{array} \quad \text { otherwise. }
\end{array}\right.
$$

This algorithm was developed by Rockafellar [23] in 1976. For a given $x^{0} \in \mathbb{R}_{+}^{n}$ and $\beta_{k} \geq \beta>0$, the iterate $x^{k+1}$ is solution to the following recursion

$$
\text { (PPA) } \quad 0 \in \beta_{k} T(x)+\nabla_{x} q\left(x, x^{k}\right),
$$

where

$$
q\left(x, x^{k}\right)=\frac{1}{2}\left\|x-x^{k}\right\|^{2}
$$

${ }^{*}$ Corresponding author.

E-mail addresses: babedallah@yahoo.com (A. Bnouhachem), qinxl@yuntech.edu.tw (X. Qin).

Received November 22, 2019; Accepted February 27, 2020. 
is a quadratic function of $x$.

It is more practical to find approximate solutions of problem (1.2) rather than the exact solutions due to the fact that this excludes some practical applications in general. Rockafellar [23] proposed to solve problem (1.2) approximately, that is, the new iterate $x^{k+1}$ satisfies

$$
e^{k} \in\left(x-x^{k}\right)+\beta_{k} T(x),
$$

where $e^{k} \in \mathbb{R}^{n}$ is the error term. Some inexact PPAs [8, 21, 24, 25] have been developed, and the condition that the error term being summable is an essential condition for the convergence of these algorithms. For example, Eckstein [11] supposed that

$$
\sum_{k=1}^{\infty}\left\|e^{k}\right\|<+\infty \quad \text { and } \quad \sum_{k=1}^{\infty}\left\langle e^{k}, x^{k}\right\rangle<+\infty .
$$

Han and He [16] proved the convergence of inexact PPA (1.4) under the assumptions

$$
\left\|e^{k}\right\| \leq v_{k}\left\|x^{k}-x^{k+1}\right\| \quad \text { and } \quad \sum_{k=0}^{\infty} v_{k}^{2}<+\infty .
$$

Recently, a number of authors have focused on the generalization of PPA by replacing the usual quadratic term (1.2) with some nonlinear functions. Auslender, Teboulle and Ben-Tiba $[1,2]$ proposed logarithmicquadratic proximal (LQP) method where a distance-like function replaces the usual quadratic term. More precisely, let $\mu \in(0,1)$ be a constant. For given $x^{k} \in \mathbb{R}_{++}^{n}$ and $\beta_{k} \geq \beta>0$, the new iterate $x^{k+1}$ is solution of the following set-valued equation:

$$
0 \in \beta_{k} T(x)+\nabla_{x} Q\left(x, x^{k}\right)
$$

where

$$
\nabla_{x} Q\left(x, x^{k}\right)=x-x^{k}+\mu X_{k} \log \frac{x}{x^{k}}
$$

and $X_{k}=\operatorname{diag}\left(x_{1}^{k}, \ldots, x_{n}^{k}\right)$ and $\log \frac{x}{x^{k}}=\left(\log \frac{x_{1}}{x_{1}^{k}}, \ldots, \log \frac{x_{n}}{x_{n}^{k}}\right)^{T}$. In order to ensure the convergence, Auslender, Teboulle and Ben-Tiba [1] suggested to use the accuracy criterion of type (1.5). Since $N_{\mathbb{R}_{+}^{n}}=\{0\}$, one has the problem (1.7) is equivalent to the following systems of nonlinear equations

$$
\beta_{k} F(x)+x-x^{k}+\mu X_{k} \log \frac{x}{x^{k}}=0 .
$$

Many practical problems show that often one of subproblem (1.8) is expensive to solve. Driven by the fact of eliminating this drawback, various iterative algorithms for approximating solutions have been proposed by numerous researchers, see, e.g., [4, 6, 5, 7, 17, 20, 26, 27]. Based on the work of [1], Bnouhachem [4] proposed that the predictor is obtained via solving the following system

$$
0 \approx \beta_{k} F\left(\tilde{x}^{k}\right)+\tilde{x}^{k}-x^{k}+\mu X_{k} \log \frac{\tilde{x}^{k}}{x^{k}}=\xi^{k}
$$

and $\xi^{k}$ satisfies

$$
\left\|\xi^{k}\right\| \leq \eta\left\|x^{k}-\tilde{x}^{k}\right\|, \quad 0<\eta<1
$$

In this paper, motivated by the results of $[4,17,27]$, we consider and analyze an LQP method for NCP. The key features of this method are the predictor is obtained by solving (1.8) approximately, with more relaxed conditions than (1.9) and the new iterate is obtained by using a new step size $\alpha_{k}$. Further, we prove that the sequence generated by the iterative scheme converges 
under certain assumptions. Finally, in order to verify the theoretical assertions, some numerical examples are given. The algorithm and results presented in this paper extend some recent corresponding algorithms and results.

\section{PRELIMINARIES}

In this section, we list some preliminaries and simple results for further analysis. First, we use $\mathbb{R}$ to represent the usual Euclidean space and $\|$.$\| denote the Euclidean norm in \mathbb{R}^{n}$.

Definition 2.1. Let $v \in \mathbb{R}^{n}$. The projection of $v$ onto $\mathbb{R}_{+}^{n}$, denoted by $P_{\mathbb{R}_{+}^{n}}[v]$, is defined by

$$
P_{\mathbb{R}_{+}^{n}}[v]:=\arg \min \left\{\|v-u\| / u \in \mathbb{R}_{+}^{n}\right\} .
$$

Definition 2.2. An operator $F: \mathbb{R}^{n} \rightarrow \mathbb{R}^{n}$ is said to be pseudomonotone if, $\forall u, v \in \mathbb{R}^{n}$,

$$
(v-u)^{T} F(u) \geq 0 \Rightarrow(v-u)^{T} F(v) \geq 0 .
$$

Lemma 2.1. From the definition of the projection, we have

1) $\forall u \in \mathbb{R}_{+}^{n}, \forall v \in \mathbb{R}^{n}$,

$$
\left(v-P_{\mathbb{R}_{+}^{n}}[v]\right)^{T}\left(u-P_{\mathbb{R}_{+}^{n}}[v]\right) \leq 0 .
$$

2) $\forall u, v \in \mathbb{R}^{n}$,

$$
\left\|P_{\mathbb{R}_{+}^{n}}[v]-P_{\mathbb{R}_{+}^{n}}[u]\right\| \leq\|v-u\|
$$

3) $\forall v \in \mathbb{R}^{n}, u \in \mathbb{R}_{+}^{n}$,

$$
\left\|P_{\mathbb{R}_{+}^{n}}[v]-u\right\|^{2} \leq\|v-u\|^{2}-\left\|v-P_{\mathbb{R}_{+}^{n}}[v]\right\|^{2} .
$$

Lemma 2.2. [4] For given $x^{k}>0$ and $q \in \mathbb{R}^{n}$, let $x$ be the positive solution of the following equation:

$$
q+x-x^{k}+\mu X_{k} \log \frac{x}{x^{k}}=0
$$

where $X_{k}=\operatorname{diag}\left(x_{1}^{k}, \ldots, x_{n}^{k}\right)$ and $\log \frac{x}{x^{k}}=\left(\log \frac{x_{1}}{x_{1}^{k}}, \ldots, \log \frac{x_{n}}{x_{n}^{k}}\right)$. Then, for any $y \geq 0$,

$$
(y-x)^{T} q \geq \frac{1+\mu}{2}\left(\|x-y\|^{2}-\left\|x^{k}-y\right\|^{2}\right)+\frac{1-\mu}{2}\left\|x^{k}-x\right\|^{2} .
$$

Lemma 2.3. (Robbins-Siegmund theorem [22]) Let $z_{k}, \rho_{k}, \xi_{k}$ and $\zeta_{k}$ be nonnegative variables such that

$$
z_{k+1} \leq\left(1+\rho_{k}\right) z_{k}+\sigma_{k}-\zeta_{k}
$$

Then $\lim _{k \rightarrow \infty} z_{k}$ exists and is finite and $\sum_{k=0}^{\infty} \zeta_{k}<\infty$ when $\sum_{k=0}^{\infty} \rho_{k}<\infty$ and $\sum_{k=0}^{\infty} \sigma_{k}<\infty$.

Proof. Define

$$
z_{k}^{\prime}=z_{k} \prod_{j=0}^{k-1}\left(1+\rho_{j}\right)^{-1}, \sigma_{k}^{\prime}=\sigma_{k} \prod_{j=0}^{k}\left(1+\rho_{j}\right)^{-1}, \text { and } \zeta_{k}^{\prime}=\zeta_{k} \prod_{j=0}^{k}\left(1+\rho_{j}\right)^{-1} \text {. }
$$

Then, (2.7) can be written as

$$
z_{k+1}^{\prime} \leq z_{k}^{\prime}+\sigma_{k}^{\prime}-\zeta_{k}^{\prime}
$$

and

$$
\sum_{k=0}^{\infty} \sigma_{k}^{\prime} \leq \sum_{k=0}^{\infty} \sigma_{k}<\infty
$$


Let

$$
u_{k}=z_{k}^{\prime}-\sum_{j=0}^{k-1}\left(\sigma_{j}^{\prime}-\zeta_{j}^{\prime}\right)
$$

Then $u_{k}>-\infty$. From (2.8), we have

$$
u_{k+1}=z_{k+1}^{\prime}-\sum_{j=0}^{k}\left(\sigma_{j}^{\prime}-\zeta_{j}^{\prime}\right) \leq z_{k}^{\prime}+\sigma_{k}^{\prime}-\zeta_{k}^{\prime}-\sum_{j=0}^{k}\left(\sigma_{j}^{\prime}-\zeta_{j}^{\prime}\right)=u_{k} .
$$

So, $\lim _{k \rightarrow \infty} u_{k}$ exists and is finite on the condition of $\sum_{k=0}^{\infty} \sigma_{k}^{\prime}$ according to the fact that sequence $\left\{u_{k}\right\}$ is monotone and bounded. Using (2.10), one has

$$
z_{k}^{\prime}=u_{k}+\sum_{j=0}^{k-1}\left(\sigma_{j}^{\prime}-\zeta_{j}^{\prime}\right) \geq 0
$$

which implies

$$
0 \leq \sum_{j=0}^{k-1} \zeta_{j}^{\prime} \leq u_{k}+\sum_{j=0}^{k-1} \sigma_{j}^{\prime}
$$

As $k$ tends to $\infty, \sum_{k=0}^{\infty} \zeta_{k}^{\prime}<\infty$ under the (2.9), one has that $\lim _{k \rightarrow \infty} z_{k}^{\prime}$ exists and is finite. Finally, according to the equivalence relation between $z_{k}$ and $z_{k}^{\prime}$

$$
z_{k}=z_{k}^{\prime} \prod_{j=0}^{k-1}\left(1+\rho_{j}\right)
$$

and the inequality

$$
\zeta_{k}=\zeta_{k}^{\prime} \prod_{j=0}^{k}\left(1+\rho_{j}\right) \leq \zeta_{k}^{\prime} \prod_{j=0}^{\infty}\left(1+\rho_{j}\right)
$$

we can get that $\lim _{k \rightarrow \infty} z_{k}$ exists and is finite and $\sum_{k=0}^{\infty} \zeta_{k}<\infty$ when $\sum_{k=0}^{\infty} \rho_{k}<\infty$ and $\sum_{k=0}^{\infty} \sigma_{k}<$ $\infty$. This completes the proof.

Lemma 2.4. [6] Let $x^{*} \in \Omega^{*}, \tilde{x}^{k} \in \mathbb{R}_{+}^{n}, 0<\tau<1,0<\mu<1$ and $x^{k+1}(\alpha)$ be defined by $x^{k+1}\left(\alpha_{k}\right)=\tau x^{k}+(1-\tau) P_{\mathbb{R}_{+}^{n}}\left[x^{k}-\frac{\alpha_{k}}{1+\mu} F\left(\tilde{x}^{k}\right)\right]$. Then

$$
\begin{aligned}
\left\|x^{k+1}\left(\alpha_{k}\right)-x^{*}\right\|^{2} \leq & \left\|x^{k}-x^{*}\right\|^{2}-(1-\tau)\left\{\left\|x^{k}-x_{p}^{k}\left(\alpha_{k}\right)\right\|^{2}\right. \\
& \left.+\frac{2 \alpha_{k}}{1+\mu}\left\langle x_{p}^{k}\left(\alpha_{k}\right)-x^{k}, F\left(\tilde{x}^{k}\right)\right\rangle+\frac{2 \alpha_{k}}{1+\mu}\left\langle x^{k}-x^{*}, F\left(\tilde{x}^{k}\right)\right\rangle\right\},
\end{aligned}
$$

where $x_{p}^{k}\left(\alpha_{k}\right)=P_{\mathbb{R}_{+}^{n}}\left[x^{k}-\frac{\alpha_{k}}{1+\mu} F\left(\tilde{x}^{k}\right)\right]$.

\section{THE ITERATIVE METHOD AND CONVERGENCE RESUlTS}

For given $x^{k}>0, \beta_{k}>0$, let $\delta_{k}$ be a sequence of positive real numbers such that $\sum_{k=1}^{\infty} \delta_{k}<\infty$. We propose the following inexact LQP method for solving NCP.

Prediction step: Find an approximate positive solution $\tilde{x}^{k}$ of (1.8), called predictor, such that

$$
0 \approx \beta_{k} F\left(\tilde{x}^{k}\right)+\tilde{x}^{k}-x^{k}+\mu X_{k} \log \frac{\tilde{x}^{k}}{x^{k}}=\xi^{k}:=\beta_{k}\left(F\left(\tilde{x}^{k}\right)-F\left(x^{k}\right)\right)
$$

and $\xi^{k}$ satisfies 


$$
\left|\left\langle x^{k}-\tilde{x}^{k}, \xi^{k}\right\rangle\right| \leq \eta\left\|x^{k}-\tilde{x}^{k}\right\|^{2}, \quad 0<\eta<1 .
$$

Correction step: For $0<\tau<1$, the new iterate $x^{k+1}\left(\alpha_{k}\right)$ is defined by

$$
x^{k+1}\left(\alpha_{k}\right)=\tau x^{k}+(1-\tau) P_{\mathbb{R}_{+}^{n}}\left[x^{k}-\frac{\alpha_{k}}{1+\mu} F\left(\tilde{x}^{k}\right)\right]
$$

where

$$
\begin{gathered}
\alpha_{k}=\beta_{k}\left(\gamma+\delta_{k}\right) \frac{\varphi_{k}}{\left\|d^{k}\right\|^{2}} \\
\varphi_{k}=\frac{1}{1+\mu}\left\|x^{k}-\tilde{x}^{k}\right\|^{2}+\frac{1}{1+\mu}\left\langle x^{k}-\tilde{x}^{k}, \xi^{k}\right\rangle
\end{gathered}
$$

and

$$
d^{k}=\left(x^{k}-\tilde{x}^{k}\right)+\frac{1}{1+\mu} \xi^{k} .
$$

Remark 3.1. Note that (3.1) is equivalent to the following system of nonlinear equations

$$
\beta_{k} F\left(x^{k}\right)+\tilde{x}^{k}-x^{k}+\mu X_{k} \log \frac{\tilde{x}^{k}}{x^{k}}=0 .
$$

Hence

$$
\tilde{x}_{j}^{k}+\mu x_{j}^{k} \log \tilde{x}_{j}^{k}+\left(\beta_{k} F_{j}\left(x^{k}\right)-x_{j}^{k}-\mu x_{j}^{k} \log x_{j}^{k}\right)=0, \quad j=1, \ldots, n .
$$

The recursion of classical Newton method for the above problem is

$$
\tilde{x}_{j}^{k}=x_{j}^{k}-\frac{\beta_{k}}{1+\mu} F_{j}\left(x^{k}\right) .
$$

The solution $\tilde{x}^{k}$ of (3.8) is positive. To avoid the non-positive value $\tilde{x}_{j}^{k}$ in the iteration process, one takes

$$
\tilde{x}^{k}:=\rho x^{k}+(1-\rho) P_{\mathbb{R}_{+}^{n}}\left[x^{k}-\frac{\beta_{k}}{1+\mu} F\left(x^{k}\right)\right], \quad 0<\rho<1 .
$$

Remark 3.2. Solving the NCP is equivalent to finding a zero point of the continuous nonsmooth function

$$
e\left(x^{k}, \beta_{k}\right):=x^{k}-P_{\mathbb{R}_{+}^{n}}\left[x^{k}-\frac{\beta_{k}}{1+\mu} F\left(x^{k}\right)\right]
$$

For any $x \in \Omega^{*}$, we let

$$
\operatorname{dist}\left(x, \Omega^{*}\right):=\min \left\{\left\|x-x^{*}\right\| / x^{*} \in \Omega^{*}\right\}
$$

It is clear that

$$
\operatorname{dist}\left(x, \Omega^{*}\right)=0 \Leftrightarrow e\left(x, \beta_{k}\right)=0 .
$$

Lemma 3.1. If $F$ is Lipschitz continuous with constant $L$, then
(i) $\left\|x^{k}-\tilde{x}^{k}\right\|^{2} \leq c\left\|x^{k}-x^{*}\right\|^{2}$, where $c=6-\rho+4 \frac{(1-\rho) \beta_{k}^{2} L^{2}}{(1+\mu)^{2}}$.
(ii) $\varphi_{k} \geq\left(\frac{1-\eta}{1+\mu}\right)\left\|x^{k}-\tilde{x}^{k}\right\|^{2}$.
(iii) $\varphi_{k} \geq \frac{(1-\eta)}{2(1+\mu)\left(1+\beta_{k}^{2} L^{2}\right)}\left\|d^{k}\right\|^{2}$. 
Proof. For any $a, b \in \mathbb{R}^{n}$, we have $\|a+b\|^{2} \leq 2\|a\|^{2}+2\|b\|^{2}$. It follows that

$$
\begin{aligned}
\left\|x^{k}-\tilde{x}^{k}\right\|^{2} \leq & 2\left\|x^{k}-x^{*}\right\|^{2}+2\left\|\tilde{x}^{k}-x^{*}\right\|^{2} \\
= & 2\left\|x^{k}-x^{*}\right\|^{2}+2 \| \rho\left(x^{k}-x^{*}\right)+(1-\rho)\left(P_{R_{+}^{n}}\left[x^{k}-\frac{\beta_{k}}{1+\mu} F\left(x^{k}\right)\right]\right. \\
& \left.-P_{R_{+}^{n}}\left[x^{*}-\frac{\beta_{k}}{1+\mu} F\left(x^{*}\right)\right]\right) \|^{2} \\
\leq & 2\left\|x^{k}-x^{*}\right\|^{2}+2 \rho\left\|x^{k}-x^{*}\right\|^{2}+2(1-\rho) \|\left(P_{R_{+}^{n}}\left[x^{k}-\frac{\beta_{k}}{1+\mu} F\left(x^{k}\right)\right]\right. \\
& \left.-P_{R_{+}^{n}}\left[x^{*}-\frac{\beta_{k}}{1+\mu} F\left(x^{*}\right)\right]\right) \|^{2} \\
\leq & 2\left\|x^{k}-x^{*}\right\|^{2}+2\left(2-\rho+2 \frac{(1-\rho) \beta_{k}^{2} L^{2}}{(1+\mu)^{2}}\right)\left\|x^{k}-x^{*}\right\|^{2} \\
= & \left(6-\rho+4 \frac{(1-\rho) \beta_{k}^{2} L^{2}}{(1+\mu)^{2}}\right)\left\|x^{k}-x^{*}\right\|^{2},
\end{aligned}
$$

where the second inequality follows from the Lipschitz continuity of $F$. Setting

$$
c=6-\rho+4 \frac{(1-\rho) \beta_{k}^{2} L^{2}}{(1+\mu)^{2}}
$$

in (3.11), we get the assertions (i) immediately.

Now, we show the assertions (ii) and (iii). It follows from (3.2) and (3.5) that

$$
\varphi_{k}=\frac{1}{1+\mu}\left\|x^{k}-\tilde{x}^{k}\right\|^{2}+\frac{1}{1+\mu}\left(x^{k}-\tilde{x}^{k}\right)^{T} \xi^{k} \geq\left(\frac{1-\eta}{1+\mu}\right)\left\|x^{k}-\tilde{x}^{k}\right\|^{2} .
$$

Moreover,

$$
\begin{aligned}
\varphi_{k} & \geq \frac{2(1-\eta)}{2(1+\mu)}\left(\frac{\beta_{k}^{2} L^{2}}{1+\beta_{k}^{2} L^{2}}\left\|x^{k}-\tilde{x}^{k}\right\|^{2}+\frac{1}{1+\beta_{k}^{2} L^{2}}\left\|x^{k}-\tilde{x}^{k}\right\|^{2}\right) \\
& \geq \frac{2(1-\eta)}{2(1+\mu)}\left(\frac{\beta_{k}^{2}}{1+\beta_{k}^{2} L^{2}}\left\|F\left(x^{k}\right)-F\left(\tilde{x}^{k}\right)\right\|^{2}+\frac{1}{1+\beta_{k}^{2} L^{2}}\left\|x^{k}-\tilde{x}^{k}\right\|^{2}\right) \\
& \geq \frac{2(1-\eta)}{2(1+\mu)}\left(\frac{\beta_{k}^{2}}{(1+\mu)^{2}\left(1+\beta_{k}^{2} L^{2}\right)}\left\|F\left(x^{k}\right)-F\left(\tilde{x}^{k}\right)\right\|^{2}+\frac{1}{1+\beta_{k}^{2} L^{2}}\left\|x^{k}-\tilde{x}^{k}\right\|^{2}\right) \\
& \geq \frac{(1-\eta)}{2(1+\mu)\left(1+\beta_{k}^{2} L^{2}\right)}\left\|\left(x^{k}-\tilde{x}^{k}\right)+\frac{1}{1+\mu} \xi^{k}\right\|^{2} \\
& =\frac{(1-\eta)}{2(1+\mu)\left(1+\beta_{k}^{2} L^{2}\right)}\left\|d^{k}\right\|^{2} .
\end{aligned}
$$

This completes the proof.

Inspired by Lemma 2.2, the contraction property of the proposed method is presented in the following theorem. 
Theorem 3.1. If $F$ is Lipschitz continuous with constant L. Let $\left\{x^{k}\right\}$ be a sequence generated by the proposed method and $x^{*}$ be a solution of NCP. Then

$$
\left\|x^{k+1}\left(\alpha_{k}\right)-x^{*}\right\|^{2} \leq\left\|x^{k}-x^{*}\right\|^{2}-(1-\tau)\left(2-\gamma-\delta_{k}\right)\left(\gamma+\delta_{k}\right) \frac{\varphi_{k}^{2}}{\left\|d^{k}\right\|^{2}}
$$

Proof. By setting $q=\beta_{k} F\left(\tilde{x}^{k}\right)-\xi^{k}$ in (2.5) and $y=x_{p}^{k}\left(\alpha_{k}\right)$ in (2.6), one has

$$
\begin{aligned}
\left\langle x_{p}^{k}\left(\alpha_{k}\right)-\tilde{x}^{k}, \frac{1}{1+\mu}\left(\xi^{k}-\beta_{k} F\left(\tilde{x}^{k}\right)\right)\right\rangle \leq & \frac{1}{2}\left(\left\|x^{k}-x_{p}^{k}\left(\alpha_{k}\right)\right\|^{2}-\left\|\tilde{x}^{k}-x_{p}^{k}\left(\alpha_{k}\right)\right\|^{2}\right) \\
& -\frac{1-\mu}{2(1+\mu)}\left\|x^{k}-\tilde{x}^{k}\right\|^{2} .
\end{aligned}
$$

Recall that

$$
\left\langle x_{p}^{k}\left(\alpha_{k}\right)-\tilde{x}^{k}, x^{k}-\tilde{x}^{k}\right\rangle=\frac{1}{2}\left(\left\|\tilde{x}^{k}-x_{p}^{k}\left(\alpha_{k}\right)\right\|^{2}-\left\|x^{k}-x_{p}^{k}\left(\alpha_{k}\right)\right\|^{2}\right)+\frac{1}{2}\left\|x^{k}-\tilde{x}^{k}\right\|^{2}
$$

Adding (3.13) and (3.14), we then obtain

$$
\left\langle x_{p}^{k}\left(\alpha_{k}\right)-\tilde{x}^{k}, x^{k}-\tilde{x}^{k}+\frac{1}{1+\mu}\left(\xi^{k}-\beta_{k} F\left(\tilde{x}^{k}\right)\right)\right\rangle \leq \frac{\mu}{1+\mu}\left\|x^{k}-\tilde{x}^{k}\right\|^{2},
$$

which implies

$$
\frac{2 \alpha_{k}}{\beta_{k}}\left\langle x_{p}^{k}\left(\alpha_{k}\right)-\tilde{x}^{k}, x^{k}-\tilde{x}^{k}+\frac{1}{1+\mu}\left(\xi^{k}-\beta_{k} F\left(\tilde{x}^{k}\right)\right)\right\rangle-\frac{2 \alpha_{k} \mu}{\beta_{k}(1+\mu)}\left\|x^{k}-\tilde{x}^{k}\right\|^{2} \leq 0 .
$$

Using Lemma 2.4, we get

$$
\begin{aligned}
\left\|x^{k+1}\left(\alpha_{k}\right)-x^{*}\right\|^{2} \leq & \left\|x^{k}-x^{*}\right\|^{2}-(1-\tau)\left\{\left\|x^{k}-x_{p}^{k}\left(\alpha_{k}\right)\right\|^{2}+\frac{2 \alpha_{k}}{1+\mu}\left\langle x_{p}^{k}\left(\alpha_{k}\right)-x^{k}, F\left(\tilde{x}^{k}\right)\right\rangle\right. \\
& \left.+\frac{2 \alpha_{k}}{1+\mu}\left\langle x^{k}-x^{*}, F\left(\tilde{x}^{k}\right)\right\rangle\right\} .
\end{aligned}
$$

Since $\tilde{x}^{k} \in \mathbb{R}_{+}^{n}$ and $x^{*}$ is a solution of NCP, using the pseudomonotonicity of $F$, we have

$$
\left\langle\tilde{x}^{k}-x^{*}, F\left(x^{*}\right)\right\rangle=\left\langle\tilde{x}^{k}, F\left(x^{*}\right)\right\rangle \geq 0 \Rightarrow\left\langle\tilde{x}^{k}-x^{*}, F\left(\tilde{x}^{k}\right\rangle \geq 0\right.
$$

and consequently

$$
\left\langle x^{k}-x^{*}, F\left(\tilde{x}^{k}\right)\right\rangle \geq\left\langle x^{k}-\tilde{x}^{k}, F\left(\tilde{x}^{k}\right)\right\rangle .
$$


Applying (3.17) to the last term in the right side of (3.16) and adding (3.15), and using the notation of $d^{k}$ in (3.6), we obtain

$$
\begin{aligned}
\left\|x^{k+1}\left(\alpha_{k}\right)-x^{*}\right\|^{2} \leq & \left\|x^{k}-x^{*}\right\|^{2}-(1-\tau)\left\{\left\|x^{k}-x_{p}^{k}\left(\alpha_{k}\right)\right\|^{2}+\frac{2 \alpha_{k}}{1+\mu}\left\langle x_{p}^{k}\left(\alpha_{k}\right)-\tilde{x}^{k}, F\left(\tilde{x}^{k}\right)\right\rangle\right\} \\
\leq & \left\|x^{k}-x^{*}\right\|^{2}-(1-\tau)\left\{\left\|x_{p}^{k}\left(\alpha_{k}\right)-x^{k}\right\|^{2}+\frac{2 \alpha_{k}}{\beta_{k}}\left\langle x_{p}^{k}\left(\alpha_{k}\right)-x^{k}, d^{k}\right\rangle\right. \\
& \left.+\frac{2 \alpha_{k}}{\beta_{k}}\left\langle x^{k}-\tilde{x}^{k}, d^{k}\right\rangle-\frac{2 \alpha_{k} \mu}{\beta_{k}(1+\mu)}\left\|x^{k}-\tilde{x}^{k}\right\|^{2}\right\} \\
= & \left\|x^{k}-x^{*}\right\|^{2}-(1-\tau)\left\{\left\|x_{p}^{k}\left(\alpha_{k}\right)-x^{k}+\frac{\alpha_{k}}{\beta_{k}} d^{k}\right\|^{2}-\frac{\alpha_{k}^{2}}{\beta_{k}^{2}}\left\|d^{k}\right\|^{2}\right. \\
& \left.+\frac{2 \alpha_{k}}{\beta_{k}}\left\langle x^{k}-\tilde{x}^{k}, d^{k}\right\rangle-\frac{2 \alpha_{k} \mu}{\beta_{k}(1+\mu)}\left\|x^{k}-\tilde{x}^{k}\right\|^{2}\right\} \\
\leq & \left\|x^{k}-x^{*}\right\|^{2}-(1-\tau)\left\{\frac{2 \alpha_{k}}{\beta_{k}}\left\langle x^{k}-\tilde{x}^{k}, x^{k}-\tilde{x}^{k}+\frac{1}{1+\mu} \xi^{k}\right\rangle\right. \\
& \left.-\frac{2 \alpha_{k} \mu}{\beta_{k}(1+\mu)}\left\|x^{k}-\tilde{x}^{k}\right\|^{2}-\frac{\alpha_{k}^{2}}{\beta_{k}^{2}}\left\|d^{k}\right\|^{2}\right\} \\
= & \left\|x^{k}-x^{*}\right\|^{2}-(1-\tau)\left\{\frac{2 \alpha_{k}}{\beta_{k}}\left(\frac{1}{1+\mu}\left\|x^{k}-\tilde{x}^{k}\right\|^{2}+\frac{1}{1+\mu}\left\langle x^{k}-\tilde{x}^{k}, \xi^{k}\right\rangle\right)\right. \\
& \left.-\frac{\alpha_{k}^{2}}{\beta_{k}^{2}}\left\|d^{k}\right\|^{2}\right\} \\
= & \left\|x^{k}-x^{*}\right\|^{2}-(1-\tau)\left(\frac{2 \alpha_{k}}{\beta_{k}} \varphi_{k}-\frac{\alpha_{k}^{2}}{\beta_{k}^{2}}\left\|d^{k}\right\|^{2}\right)
\end{aligned}
$$

From the definition of $\alpha_{k}$, we obtain that

$$
\left\|x^{k+1}\left(\alpha_{k}\right)-x^{*}\right\|^{2} \leq\left\|x^{k}-x^{*}\right\|^{2}-(1-\tau)\left(2-\gamma-\delta_{k}\right)\left(\gamma+\delta_{k}\right) \frac{\varphi_{k}^{2}}{\left\|d^{k}\right\|^{2}}
$$

The conclusion of this theorem is proved.

With Lemma 3.1 and Theorem 3.2 at hand, we are able to prove the convergence of the proposed method.

Theorem 3.2. Let $\left\{x^{k}\right\}$ be a sequence generated by the proposed method and $x^{*}$ be a solution of NCP. Then, we have

$$
\left\|x^{k+1}\left(\alpha_{k}\right)-x^{*}\right\|^{2} \leq\left(1+(1-\tau) c \delta_{k}\left(\gamma+\delta_{k}\right)\right)\left\|x^{k}-x^{*}\right\|^{2}-(1-\tau)(2-\gamma)\left(\gamma+\delta_{k}\right) \frac{\varphi_{k}^{2}}{\left\|d^{k}\right\|^{2}}
$$

and the proposed method is convergent in the sense $\lim _{k \rightarrow \infty} \operatorname{dist}\left(x^{k}, \Omega^{*}\right)=0$. 
Proof. $\quad$ Using Lemme 3.1 (i), we obtain

$$
\begin{aligned}
\left\|x^{k+1}\left(\alpha_{k}\right)-x^{*}\right\|^{2} \leq & \left\|x^{k}-x^{*}\right\|^{2}-(1-\tau)(2-\gamma)\left(\gamma+\delta_{k}\right) \frac{\varphi_{k}^{2}}{\left\|d^{k}\right\|^{2}} \\
& +(1-\tau) \delta_{k}\left(\gamma+\delta_{k}\right) \frac{\varphi_{k}^{2}}{\left\|d^{k}\right\|^{2}} \\
\leq & \left\|x^{k}-x^{*}\right\|^{2}-(1-\tau)(2-\gamma)\left(\gamma+\delta_{k}\right) \frac{\varphi_{k}^{2}}{\left\|d^{k}\right\|^{2}} \\
& +(1-\tau) \delta_{k}\left(\gamma+\delta_{k}\right) \frac{\left(\left\langle\tilde{x}^{k}-x^{k}, d^{k}\right\rangle\right)^{2}}{\left\|d^{k}\right\|^{2}} \\
\leq & \left\|x^{k}-x^{*}\right\|^{2}-(1-\tau)(2-\gamma)\left(\gamma+\delta_{k}\right) \frac{\varphi_{k}^{2}}{\left\|d^{k}\right\|^{2}} \\
& +(1-\tau) \delta_{k}\left(\gamma+\delta_{k}\right)\left\|\tilde{x}^{k}-x^{k}\right\|^{2} \\
\leq & \left(1+(1-\tau) c \delta_{k}\left(\gamma+\delta_{k}\right)\right)\left\|x^{k}-x^{*}\right\|^{2}-(1-\tau)(2-\gamma)\left(\gamma+\delta_{k}\right) \frac{\varphi_{k}^{2}}{\left\|d^{k}\right\|^{2}}
\end{aligned}
$$

and the first conclusion is proved. We now establish the proof of the second assertion. It follows from Lemma 3.1 and Theorem 3.2 that there is a constant

$$
v:=\frac{\left(\gamma+\delta_{k}\right)(2-\gamma)(1-\tau)(1-\eta)^{2}}{2(1+\mu)^{2}\left(1+\beta_{k}^{2} L^{2}\right)}>0
$$

such that

$$
\left\|x^{k+1}-x^{*}\right\|^{2} \leq\left(1+(1-\tau) c \delta_{k}\left(\gamma+\delta_{k}\right)\right)\left\|x^{k}-x^{*}\right\|^{2}-v\left\|x^{k}-\tilde{x}^{k}\right\|^{2} .
$$

Setting $z_{k+1}=\left\|x^{k+1}-x^{*}\right\|^{2}, z_{k}=\left\|x^{k}-x^{*}\right\|^{2}, \rho_{k}=(1-\tau) c \delta_{k}\left(\gamma+\delta_{k}\right), \sigma_{k}=0$ and $\zeta_{k}=v \| x^{k}-$ $\tilde{x}^{k} \|^{2}$ in (2.7), one finds that Lemma 2.3 asserts that $\left\{z_{k}\right\}$ is convergent and $\sum_{k=1}^{\infty} \zeta_{k}<\infty$. Due to the convergence of $\left\{z_{k}\right\}$, one can obtain that the sequence $\left\{x^{k}\right\}$ is bounded. Since $\sum_{k=1}^{\infty} \zeta_{k}<\infty$, we have

Consequently,

$$
\lim _{k \rightarrow \infty}\left\|x^{k}-\tilde{x}^{k}\right\|=0
$$

$$
\lim _{k \rightarrow \infty}\left\|e\left(x^{k}, \beta_{k}\right)\right\|=0 .
$$

Then there exists a bounded closed set, say $S\left(x^{0}\right)$, such that $\left\{x^{k}\right\} \subset S\left(x^{0}\right)$. If

$$
\limsup _{k \rightarrow \infty} \operatorname{dist}\left(x^{k}, \Omega^{*}\right)=\delta>0,
$$

then

$$
\left\{x^{k}\right\} \subset S=S\left(x^{0}\right) \cap\left\{x \mid \operatorname{dist}\left(x, \Omega^{*}\right) \geq \delta / 2\right\} .
$$

Since $S \cap \Omega^{*}=\emptyset$, one has that $e\left(x, \beta_{k}\right) \neq 0$ for all $x \in S$. Since $S$ is compact and $e\left(x, \beta_{k}\right)$ is continuous on $S$, one has

$$
\min _{x \in S}\left\|e\left(x, \beta_{k}\right)\right\|=\varepsilon>0
$$

This contradicts the fact

$$
\left\{x^{k}\right\} \subset S \quad \text { and } \quad \lim _{k \rightarrow \infty}\left\|e\left(x^{k}, \beta_{k}\right)\right\|=0 .
$$

Therefore the proof is complete. 


\section{Computational Results}

In this section, we consider two examples and report our preliminary computational results which show an improvement than the earlier existing methods.

Example 4.1. We consider the nonlinear complementarity problem:

$$
x \geq 0, \quad F(x) \geq 0, \quad x^{T} F(x)=0,
$$

where

$$
F(x)=D(x)+M x+q,
$$

$D(x)$ and $M x+q$ are the nonlinear part and linear part of $F(x)$ respectively.

The matrix $M=A^{T} A+B$ is computed as follows. $A$ is a $n \times n$ matrix whose entries are randomly generated in the interval $(-5,+5)$ and the skew-symmetric matrix $B$ is generated in the same way. The components of $D(x)$ are $D_{j}(x)=d_{j} * \arctan \left(x_{j}\right)$ and $d_{j}$ is chosen randomly in $(0,1)$.

In all tests, we take the logarithmic proximal parameter $\mu=0.1, \rho=\tau=0.01$ and all iterations start with $x^{0}=(1, \ldots, 1)^{T}$ and $\beta_{0}=1$. The stopping criterion is set to be

$$
\frac{\|\min \{x, F(x)\}\|_{\infty}}{\left\|\min \left\{x^{0}, F\left(x^{0}\right)\right\}\right\|_{\infty}} \leq 10^{-7} .
$$

All codes are written in Matlab. We test the problems (4.1) with different dimensions and $q \in(-500,500)$ in Table 1 , and $q \in(-500,0)$ in Table 2 . We compare the proposed method with those in $[7,27]$. In all tests, we take $\eta=0.9, \gamma+\delta_{k}=3.35$. The test results for problems (4.1) are reported in tables 1-2. $k$ is the number of iterations and $l$ denotes the number of evaluations of mapping $F$.

TABLE 1. The numerical results for problem (4.1) with $q \in(-500,500)$

\begin{tabular}{|c||c|c|c||c|c|c||c|c|c||}
\hline \multicolumn{1}{|c||}{$\begin{array}{c}\text { Dimension of } \\
\text { the problem }\end{array}$} & \multicolumn{3}{c||}{ The proposed method } & \multicolumn{3}{c||}{ The method in [7] } & \multicolumn{3}{c||}{ The method in [27] } \\
\cline { 2 - 10 } & $\mathrm{k}$ & 1 & $\mathrm{CPU}(\mathrm{Sec})$. & $\mathrm{k}$ & $\mathrm{l}$ & $\mathrm{CPU}(\mathrm{Sec})$. & $\mathrm{k}$ & 1 & $\mathrm{CPU}(\mathrm{Sec})$. \\
\hline 200 & 110 & 254 & 0.014 & 173 & 385 & 0.049 & 275 & 591 & 0.056 \\
\hline 300 & 107 & 245 & 0.018 & 188 & 419 & 0.071 & 304 & 649 & 0.082 \\
\hline 400 & 115 & 262 & 0.028 & 191 & 425 & 0.103 & 300 & 642 & 0.127 \\
\hline 500 & 127 & 291 & 0.051 & 208 & 462 & 0.165 & 333 & 713 & 0.210 \\
\hline 700 & 113 & 261 & 0.101 & 196 & 436 & 0.294 & 321 & 687 & 0.401 \\
\hline 1000 & 105 & 247 & 0.321 & 187 & 415 & 0.790 & 302 & 647 & 1.188 \\
\hline
\end{tabular}


TABLE 2. The numerical results for problem (4.1) with $q \in(-500,0)$

\begin{tabular}{|c||c|c|c||c|c|c||c|c|c||}
\hline \multicolumn{1}{|c||}{$\begin{array}{c}\text { Dimension of } \\
\text { the problem }\end{array}$} & \multicolumn{3}{c||}{ The proposed method } & \multicolumn{3}{c||}{ The method in [7] } & \multicolumn{3}{c||}{ The method in [27] } \\
\cline { 2 - 10 } & $\mathrm{k}$ & 1 & $\mathrm{CPU}($ Sec. $)$ & $\mathrm{k}$ & 1 & $\mathrm{CPU}($ Sec. $)$ & $\mathrm{k}$ & 1 & CPU(Sec.) \\
\hline 200 & 193 & 426 & 0.037 & 337 & 746 & 0.073 & 522 & 1118 & 0.089 \\
\hline 300 & 170 & 387 & 0.058 & 329 & 729 & 0.096 & 515 & 1103 & 0.130 \\
\hline 400 & 237 & 534 & 0.112 & 412 & 909 & 0.160 & 631 & 1351 & 0.215 \\
\hline 500 & 201 & 456 & 0.151 & 417 & 919 & 0.217 & 666 & 1424 & 0.301 \\
\hline 700 & 191 & 434 & 0.310 & 362 & 801 & 0.411 & 587 & 1257 & 0.584 \\
\hline 1000 & 215 & 492 & 0.985 & 391 & 863 & 1.491 & 610 & 1303 & 2.127 \\
\hline
\end{tabular}

Tables 1-2 show the high efficiency and robustness of the proposed method. Numerical results indicate that the proposed method can be save about $51 \sim 101$ percent of the number of iterations and about $57 \sim 107$ of the amount of computing the value of function $F$ for that in [7] and about $132 \sim 212$ percent of the number of iterations and about $150 \sim 231$ of the amount of computing the value of function $F$ for that in [27].

Example 4.2. We consider a network [17] shown in Figure 1, which consists of 25 nodes, 37 links and $6 \mathrm{O} / \mathrm{D}$ pairs. We apply the proposed method in this traffic network equilibrium problems with two modifications, we use the same notations as [17]. The traffic equilibrium problems can be described as follows:

$$
x^{*} \geq 0 \quad\left(x-x^{*}\right)^{T} F\left(x^{*}\right) \geq 0, \quad \forall x \geq 0,
$$

where

$$
F(x)=A t\left(A^{T} x\right)-B \lambda\left(B^{T} x\right) .
$$

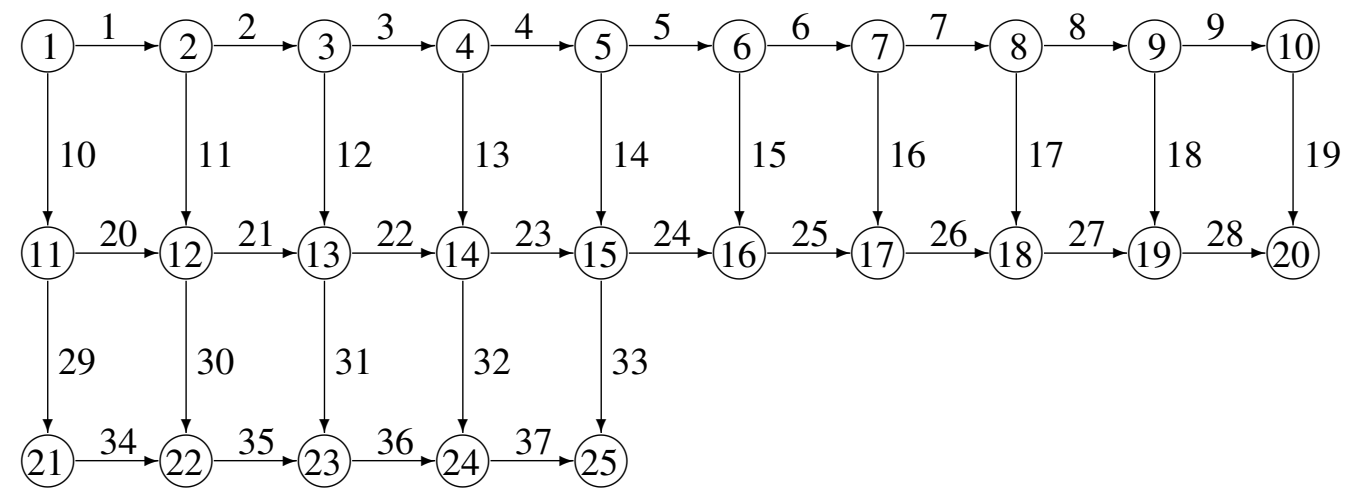

FIGURE 1. A directed network with 25 nodes and 37 links

The test results for problems (4.2) for different $\varepsilon$ are reported in Table 3, $k$ is the number of iterations and $l$ denotes the number of evaluations of mapping $F$. The stopping criterion is

$$
\frac{\|\min \{x, F(x)\}\|_{\infty}}{\left\|\min \left\{x^{0}, F\left(x^{0}\right)\right\}\right\|_{\infty}} \leq \varepsilon
$$

Table 3 reports the comparison between the methods of $[7,27]$ and the proposed method. The number of iterations and the amount of computing the value of function $F$ have great advantages. 
TABLE 3. Numerical results for for different $\varepsilon$

\begin{tabular}{|c||c|c|c||c|c|c||c|c|c||}
\hline \multicolumn{1}{|c||}{ Different } & \multicolumn{3}{c||}{ The proposed method } & \multicolumn{3}{c||}{ The method in [7] } & \multicolumn{3}{c||}{ The method in [27] } \\
\cline { 2 - 10 }$\varepsilon$ & $\mathrm{k}$ & 1 & CPU(Sec.) & $\mathrm{k}$ & $\mathrm{l}$ & $\mathrm{CPU}($ Sec.) & $\mathrm{k}$ & $\mathrm{l}$ & CPU(Sec.) \\
\hline $10^{-5}$ & 97 & 260 & 0.011 & 179 & 403 & 0.033 & 361 & 767 & 0.037 \\
\hline $10^{-6}$ & 122 & 328 & 0.013 & 231 & 523 & 0.046 & 467 & 993 & 0.049 \\
\hline $10^{-7}$ & 146 & 392 & 0.014 & 279 & 633 & 0.042 & 575 & 1223 & 0.062 \\
\hline $10^{-8}$ & 173 & 464 & 0.022 & 330 & 750 & 0.055 & 684 & 1455 & 0.077 \\
\hline $10^{-9}$ & 193 & 520 & 0.023 & 380 & 865 & 0.054 & 790 & 1680 & 0.082 \\
\hline
\end{tabular}

\section{THE CONCLUSION}

In this paper, we suggested and analyzed a new LQP method for solving NCP which unify the basic ideas of the the original the LQP method. We proposed to solve (1.8) approximately via a constructive accuracy criterion. In order to ensure the efficiency of the proposed method, we enlarged the step size in the correction step. Our results could be viewed as significant extensions of the previously known results. Finally, some numerical results are included to illustrate the efficiency of the proposed method.

\section{Acknowledgments}

The second author was supported by the Feng Tay project of National Yunlin University of Science and Technology, Taiwan. The authors are grateful to the referees for useful suggestions which improved this paper.

\section{REFERENCES}

[1] A. Auslender, M. Teboulle, S. Ben-Tiba, A Logarithmic-quadratic proximal method for variational inequalities, Comput. Optim. Appl. 12 (1999), 31-40.

[2] A. Auslender, M. Teboulle, S. Ben-Tiba, Interior proximal and multiplier methods based on second order homogenous kernels, Math. Oper. Res. 24 (1999), 646-668.

[3] A. Auslender, M. Teboule, Interior projection-like methods for monotone variational inequalities. Math. Program. Ser. A 104 (2005) 39-68.

[4] A. Bnouhachem, An LQP Method for pseudomonotone variational inequalities, J. Global Optim. 36 (2006), 351-363.

[5] A. Bnouhachem, X. Yuan, An extended LQP method for monotone nonlinear complementarity problems, J. Optim. Theory Appl. 135 (2007), 343-353.

[6] A. Bnouhachem, M.A. Noor, M. Khalfaoui, S. Zhaohan, A new logarithmic-quadratic proximal method for nonlinear complementarity problems. Appl. Math. Comput. 215 (2009), 695-706.

[7] A. Bnouhachem, A. Ou-yassine, M.A. Noor, G.Lakhnati, Modified LQP method with a new search direction for nonlinear complimentarity problems, Appl. Math. Inf. Sci. 10 (2016), 1375-1383.

[8] R. S. Burachik, A. N. Iusem, B. F. Svaiter, Enlargement of monotone operators with applications to variational inequalities, Set-Valued Anal. 5 (1997), 159-180.

[9] R. S. Burachik, A. N. Iusem, A generalized proximal point algorithm for the variational inequality problem in a Hilbert space. SIAM J. Optim. 8 (1998), 197-216.

[10] X. Dong, X. Cai, D. Han, Prediction-correction method with BB step sizes, Frontiers Math. China 13 (2018), $1325-1340$.

[11] J. Eckestein, Approximate iterations in Bregman-function-based proximal algorithms, Math. Program. 83 (1998), 113-123.

[12] M.C. Ferris, J.S. Pang, Engineering and economic applications of complementariry problems, SIAM Rev. 39 (1997), 669-713. 
[13] A. Fischer, Solution of monotone complementarity problems with locally Lipschitzian functions, Math. Program. 76 (1997), 513-532.

[14] R. Glowinski, Numerical Methods for Nonlinear Variational Inequality Problems, Springer Verlag, New York, 1984.

[15] O. Guler, On the Convergence of the proximal point algorithm for convex minimization, SIAM J. Control. Optim. 29 (1991), 403-419.

[16] D. R. Han, B. S. He, A new accuracy criterion for approximate proximal point algorithms, J. Math. Anal. Appl. 263 (2001), 343-354.

[17] B.S. He, L.Z. Liao, X.M. Yuan, A LQP based interior prediction-correction method for nonlinear complementarity problems, J. Comput. Math. 24 (2006), 33-44.

[18] B. Martinet, Régularization d'inéquations variationnelles par approximations successives, Rev. Francaise d'Informat. Recherche Opérationelle 4 (1970), 154-159.

[19] A. Nagurney and D. Zhang, Projected Dynamical Systems and Variational Inequalities with Applications, Kluwer Academic Publishers, Boston, Dordrecht, London, 1996.

[20] M. A. Noor, A. Bnouhachem, Modified proximal-point method for nonlinear complementarity problems, J. Comput. Appl. Math. 197 (2006), 395-405.

[21] J.S. Pang, Complementarity problems, in Handbook of Global Optimization, pp. 271-338, Kluwer Academic Publishers, Boston, 1995.

[22] H. Robbins, D. Siegmund, A convergence theorem for non negative almost supermartingales and some applications, In: Optimizing Methods in Statistics, pp. 233-257. Academic Press, New York, 1971.

[23] R. T. Rockafellar, Monotone Operators and the Proximal Point Algorithm, SIAM J. Control. Optim. 14 (1976), 877-898.

[24] M. V. Solodov, B. F. Svaiter, An inexact hybrid general proximal point algoritm and some new result on the theory of Bregman functions, Math. Oper. Res. 25 (2000), 214-230.

[25] M. V. Solodov, B. F. Svaiter, Error bounds for proximal point subproblems and associated inexact proximal point algorithms, Math. Program. Ser. B 88 (2000), 371-389.

[26] Y. Xu, B.S. He, X. Yuan, A hybrid inexact Logarithmic-quadratic proximal method for nolinear complementarity problems, J. Math. Anal. Appl. 322 (2006), 276-287.

[27] X. M. Yuan, The prediction-correction approach to nonlinear complementarity problems, Eur. J. Oper. Res. 176 (2007), 1357-1370. 